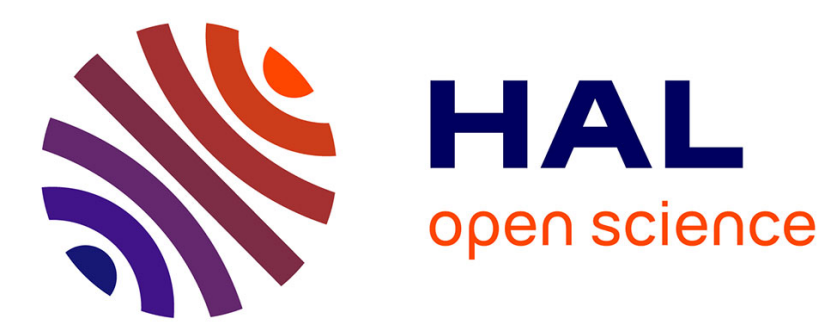

\title{
Flexible b-spline model parameterization designed for reflection tomography
}

Thimothé Perdrizet, Delphine Sinoquet

\section{To cite this version:}

Thimothé Perdrizet, Delphine Sinoquet. Flexible b-spline model parameterization designed for reflection tomography. EAGE/SEG Research Workshop, Aug 2003, Trieste, Italy. hal-02284229

\section{HAL Id: hal-02284229 \\ https://hal-ifp.archives-ouvertes.fr/hal-02284229}

Submitted on 11 Sep 2019

HAL is a multi-disciplinary open access archive for the deposit and dissemination of scientific research documents, whether they are published or not. The documents may come from teaching and research institutions in France or abroad, or from public or private research centers.
L'archive ouverte pluridisciplinaire HAL, est destinée au dépôt et à la diffusion de documents scientifiques de niveau recherche, publiés ou non, émanant des établissements d'enseignement et de recherche français ou étrangers, des laboratoires publics ou privés. 


\title{
Z-99 Flexible b-spline model parameterization designed for reflection tomography
}

Institut Français du Pétrole, $1 \& 4$ avenue de Bois-Préau 92852 Rueil-Malmaison Cedex - France

\begin{abstract}
Reflection tomography is an efficient method to determine a subsurface velocity model that best fits the traveltime data associated to the main events picked on the seismic sections. A careful choice of the model representation has to be done: a blocky model representation based on regular gridded bspline functions has been proposed. This flexible parameterization allows accurate and robust inversion but can lead to a huge number of parameters. An adaptive parameterization that enables to account for local complexities and inhomogeneous ray coverage is considered.
\end{abstract}

\section{Introduction}

Reflection tomography allows to determine a subsurface velocity model from the traveltimes associated with the main reflections of the seismic waves. Solving the forward and inverse problems requires a careful choice of the model parameterization: it affects the computation cost for the ray tracing and has strong consequences on the robustness of the inversion. The KIM consortium proposed a blocky model representation (Clarke, 1996) based on b-spline functions: its effectiveness has been proven on several applications on complex data (Ehinger et al., 2001). Nevertheless, the use of regular grids to define b-spline functions leads often to the inversion of useless parameters, especially in case of models with local complexities or with inhomogeneous ray coverage. Several authors have developed adaptive model parameterizations for tomography: Böhm et al. (2000) and Cox and Verschuur (2001) have proposed automatic data-driven adjustments of Delaunay triangle parameterization. The purpose of our work is to provide an adaptive model parameterization, based on b-spline functions, in order to limit the number of inverted parameters and consequently to make the inversion more efficient.

\section{Formulation of reflection tomography}

The forward problem of our reflection tomography (Jurado et al., 1996) is a two-point ray tracing based on a bending method: complex kinematics (converted waves, multi-valued arrivals, multiple reflections but also direct arrivals) associated with arbitrary acquisition surveys (classic surface data, OBC, VSP, walk away) can be modeled. The inverse problem consists in the minimization of a least-square objective function $C(m)=\left\|T^{\text {cal }}(m)-T^{\text {obs }}\right\|^{2}+\varepsilon\left\|D^{2}\left(m-m^{\text {prior }}\right)\right\|^{2}$ where $T^{\text {cal }}(m)$ represents the calculated traveltimes for the model $\mathrm{m}, T^{\text {obs }}$ denotes the observed traveltimes. $\left\|D^{2}\left(m-m^{\text {priori }}\right)\right\|^{2}$ is a regularization term, composed of the sum of second derivatives of the interface depths and velocity variations. This penalization term is necessary to stabilize the inversion and allows the introduction of a priori information.

In our approach, the subsurface velocity is represented by a blocky model with interfaces described by 2D explicit b-spline functions and layer velocities described by 2D or 3D explicit b-spline functions. Constant anisotropy parameters (assuming transverse isotropy) in each layer can also be inverted (see Broto and Ehinger, 2002). The choice of explicit functions and regular discretization grids is crucial for fast ray-tracing calculations. In order to easily handle the model changes during the inversion process, we define the interfaces and the velocities on the whole definition domain of the model, creating then virtual parts of interfaces or velocities (virtual meaning non-physical). Note that this 
parameterization can be used to represent hybrid models composed of velocity blocks delimited by reflectors, and of smoothly varying velocities with embedded reflectors. Examples of complex solution models found by tomography applied on real data are displayed in Figure 1.

A realistic 3D model may require up to 50000 b-spline parameters. Delbos et al. (2001) have developed sophisticated optimization techniques for the inversion of big models which allow the introduction of linear constraints on the model. An other way to improve the efficiency of the inversion of such models is to work on the parameterization itself to limit the number of parameters.

The parameterization described above requires the a priori choice of a discretization step of the bspline functions: for instance, the ideal discretization step of the interface represented on figure 2 would be small in the complex zones and large in the simple areas (figure 2 right). Such an adaptive parameterization allows to drastically reduce the number of parameters used to describe the interface (compared to a regular grid, figure 2 left). More generally, the approach we propose here aims at reducing the inverted model size by removing from the inversion the useless, that is to say the nonphysical (e.g. virtual parts of reflectors), non-illuminated, or redundant b-spline parameters to improve the stability of the inversion, and to reduce both memory use and computation costs.

\section{Macro-parameter methodology}

To reach these objectives, we introduce macro-parameters, which are linear combinations of model parameters. Defining macro-parameters results in replacing many useless parameters (non-physical, non-illuminated, or redundant information in simple areas) by few macro-parameters to obtain a reduced sized model. In this way, an irregular parameterization can be obtained from the dense discretization (figure 2, left) by replacing one or several groups of parameters in basic macroparameters as constant values or planes (i.e. constant or linear extrapolation to deal with virtual parts of the model or unilluminated areas), or in more sophisticated ones as the merging of these parameters in one of their embedded b-spline parameterization (to deal with local complexities).

The choice whether to replace a group of parameters by a macro-parameter or not, must be carried out periodically in the inversion procedure to take into account model variations. It can be done automatically through the computation of update indicators. These indicators estimate the coarser bspline discretization that explains the data complexity, and are classically based on the resolution matrix analysis, or some related approaches, which are often expensive and a posteriori methods (Böhm et Al., 2000 and Cox and Verschuur, 2001). We are currently developing a cheaper indicator (based on the gradients), that gives the optimal a priori b-spline discretization to fit the data (as illustrated in the next sections).

\section{Application to realistic synthetic data}

The method described in the previous section is here applied on a realistic synthetic model composed of 8 interfaces and 3 velocities, including a smooth (3D) velocity located under the second interface (figure 4). The six deepest interfaces (3024 parameters) and the 3D velocity (6000 parameters) are inverted simultaneously with the classic and macro-parameter version of the inversion software. The deepest interfaces are only partly illuminated. We replace the many b-spline parameters in the nonilluminated zones (hatched zones on the figure 3) by macro-parameters describing simple planes. Besides, the 3D velocity is defined all over the model, even if it only applies to the volume located under the second interface, and is not illuminated beneath the deepest interface $(4 \mathrm{~km})$. We thus replace the b-spline parameters located above $1 \mathrm{~km}$ and below $4 \mathrm{~km}$ by constant macro-parameters. The inversion with macro-parameters furnishes (figure 4) very satisfactory results (RMS $=0.8 \mathrm{~ms}$ ), with a reduced number of model parameters (figure 5). These results are equivalent to the one obtained with the classic version, but with significant benefits: the macro-parameter version requires less memory, and is $25 \%$ faster. Moreover, the tuning of the regularization weights is considerably simplified, because of the introduction, via macro-parameters, of hard constraints in the areas of poor ray coverage, which may be seen as an induced (model intrinsic) regularization. 


\section{Application to a 2D model with local complexities}

To illustrate the effectiveness of our update indicator we now apply our method to synthetic data calculated on a model composed of a velocity and a locally complex interface. We compute the gradient-based indicator for the interface of the initial model (plane interface at $\mathrm{z}=800 \mathrm{~m}$ and constant velocity) in order to determine the b-spline discretization step required to fit the data (figure 6). A discretization step is eligible if its indicator is near zero. We notice that the interface is locally complex especially from 6 to 10 kilometers where a discretization step of 78 meters is required. The classic version is consequently performed with this step, leading to the inversion of $131 \mathrm{~b}$-spline parameters. However, between 0 and 6 kilometers, which is relatively simple region, it is possible to choose a discretization step of 156 meters (312 meters is not good enough). We have run the macroparameter version to invert the data with the chosen discretization step: 156 meters between 0 and 6 kilometers and 78 meters between 6 and 10 kilometers. The inversion is performed with 95 parameters instead of 131. The solution model (figure 7) obtained with this parameterization is equivalent to the one obtained with the classic version but with a significant gain $(27 \%)$ on the number of inverted parameters.

\section{Conclusions}

We have developed a new adaptive parameterization approach, based on an original formalism, that makes our reflection tomography even more efficient. Our model description now combines the efficiency of regular parameterization for ray-tracing and the flexibility of irregular grids for inversion. The results obtained with the macro-parameter approach compared to the classic version of the software show multiple benefits: the inversion is more stable and less sensitive to regularization weights settings (this is mainly due to the model intrinsic regularization), the computing costs and memory use are significantly reduced. This method is mainly automatic thanks to the computation of appropriate update indicators. Then, macro-parameters coupled with adequate update indicators allows an adaptive parameterization for reflection tomography. This formalism may also be used for the introduction of hard a priori constraints on the model for underdetermined problems.

\section{References}

Böhm, G., Galuppo P. and Versnaver A., 2000, 3D adaptive tomography using Delaunay triangles and Voronoi polygons, Geophys. Prospect., 48, 723-744.

Broto, K. and Ehinger, A., 2002, Anisotropic traveltime tomography for depth consistent imaging of PP and PS data, The Leading Edge, Nov.2002.

Clarke, R. A., 1996, Raytracing and tomography friendly geological models : KIM 1996 Annual Report, Institut Français du Pétrole, France. http://consortium.ifp.fr/KIM.

Cox, B.E., and Verschuur, D.J.E., 2001, Data-driven tomographic inversion of focusing operators : $71^{\text {st }}$ Ann. Internat. Mtg., Soc Expl. Geophys., Expanded Abstracts.

Delbos, F., Sinoquet, D., Gilbert, J. Ch., and Masson, R., 2001, Trust-region Gauss-Newton method for reflection tomography : KIM 2001 Annual Report, Institut Français du Pétrole. http://consortium.ifp.fr/KIM.

Ehinger, A., Broto, K., Jardin, A., and the KIMASI team, 2001, 3D tomographic velocity model determination for two North Sea case studies : $63^{\text {rd }}$ Conference and Technical Exhibition, EAGE, Expanded Abstracts.

Jurado, F., Sinoquet, D., and Ehinger, A., 1996, 3D reflection tomography designed for complex structures : $66^{\text {th }}$ Ann. Internat. Mtg., Soc Expl. Geophys., Expanded Abstracts, 711- 714.

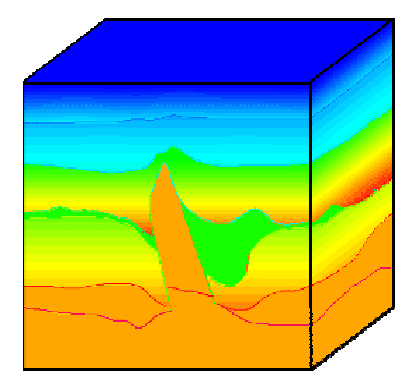

Figure 1 : Examples of 3D models determined by reflection tomography. It illustrates the flexibility of the chosen model representation. 

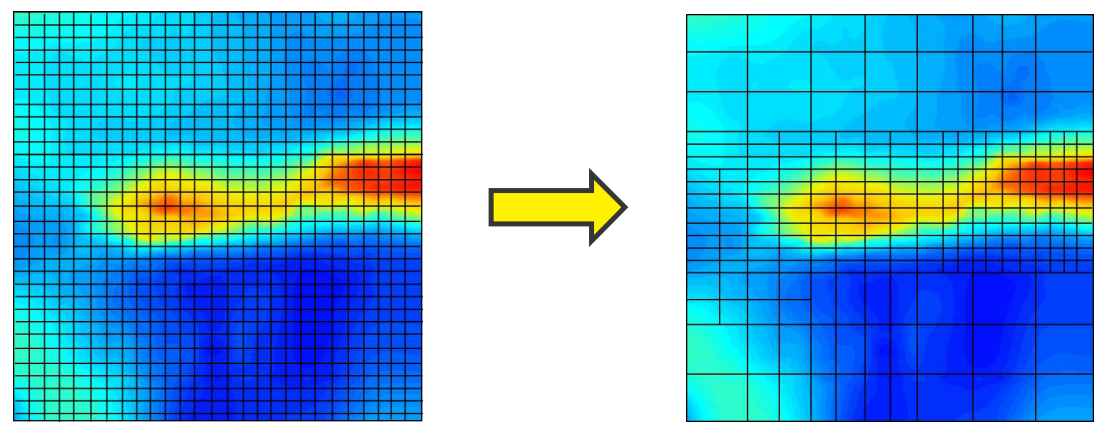

Figure 2: Regular versus irregular parameterization of an interface with local complexities. Many parameters on the left are useless and are not worth to be inverted.
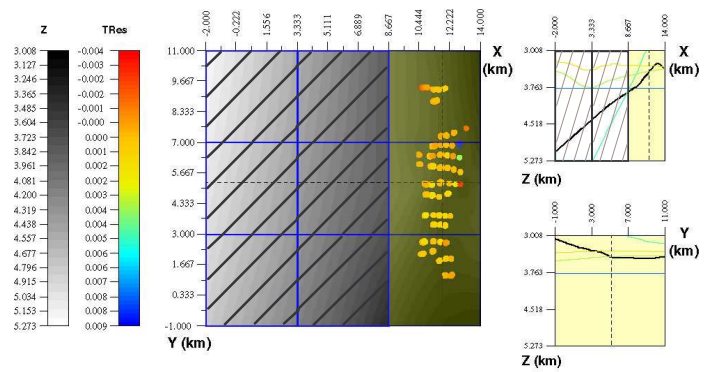

Figure 3: Map of a partly illuminated interface (hatched areas are not illuminated). It is described by 186 parameters instead of 504 .
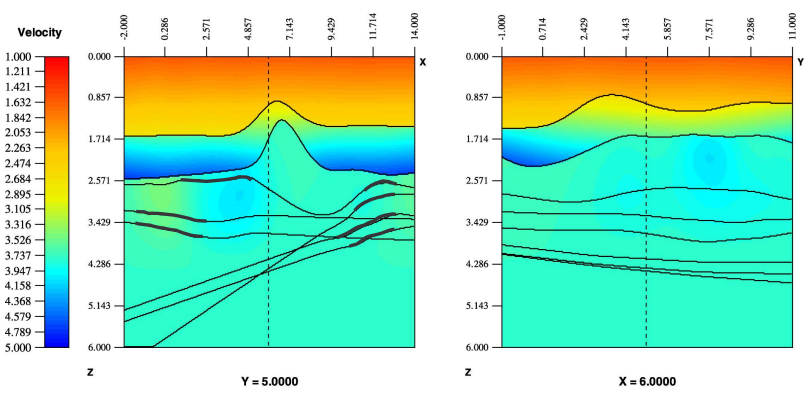

Figure 4: Solution model obtained by the macroparameter version. The bold lines correspond to the illuminated parts of the interfaces.

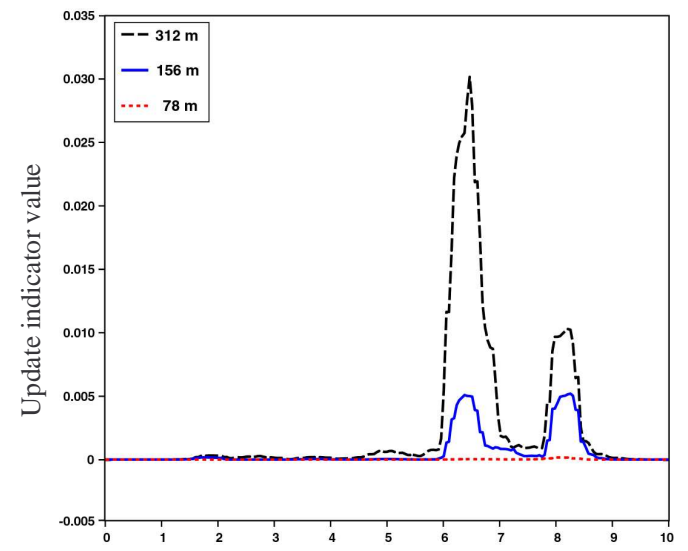

Figure 6: Update indicator. The discretization step that best fits the data gives a near-zero indicator.

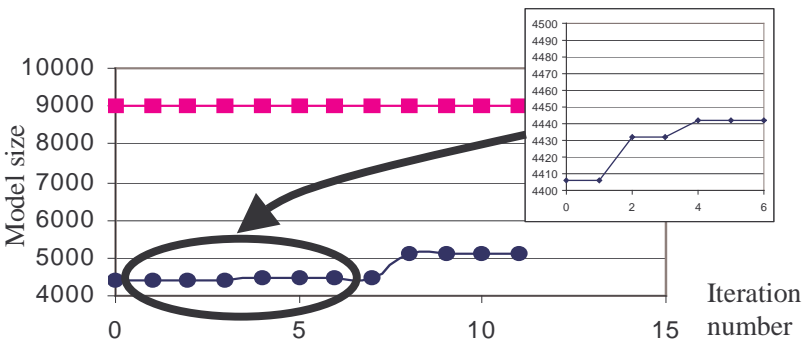

Figure 5: Inverted model size for the macroparameter (circles) and classic (squares) versions as a function of tomography iterations.

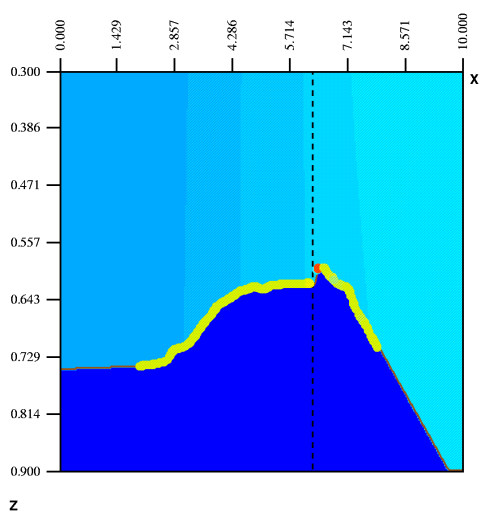

Figure 7: Solution model obtained by the macroparameter version with two different b-spline discretization steps. 